\title{
OBSERVACIONES A LA ACTUAL CONFIGURACIÓN DEL PROCESO DE TRABAJO DOCENTE EN LA EDUCACIÓN SUPERIOR
}

\author{
Aportes desde la experiencia sindical y la producción de cátedra, \\ a las nociones de subjetividad y trabajo docente en la Educación \\ Superior. La cultura académica tras la agenda del mercado
}

Gustavo Ariel Brufman (UNR)*

\begin{abstract}
Resumen
Los últimos quince años de trabajo en nuestra cátedra del Núcleo Antropológico Educativo de la carrera de Ciencias de la Educación de la UNR, su relación con el plan de estudios, y la responsabilidad sindical asumida primero como Secretario Gremial de nuestra Asociación Gremial de Docentes e Investigadores de la UNR -COAD- (20052007) y luego como Secretario General (2007-2009); motivó la necesidad de sistematizar de manera aproximativa, un conjunto de aportes conceptuales construidos con la perspectiva teórico-metodológica de un trabajo de campo de carácter etnográfico. La meta del presente artículo es introducir a la identificación de algunos de los cambios operados en el proceso de trabajo docente universitario a partir de nuevas regulaciones producidas por el modelo neoliberal demandado por el Banco Mundial para la educación superior, que han ido modificando la noción misma de trabajo docente, su organización y la cultura académica, impactando en la subjetividad del docente universitario como trabajador, en su triple condición de asalariado, profesional e intelectual desplegada en el contexto de la mercantilización y enajenación del conocimiento.
\end{abstract}

\section{Palabras Clave}

Trabajo - Subjetividad - Cultura - Flujo - Complejidad.

\begin{abstract}
The work for the last fifteen years in our chair of the Educational-Anthropological Nucleus of the Educational Sciences career at Universidad Nacional de Rosario (UNR), its relationship with the curriculum, and the trade union responsibility undertaken first as
\end{abstract}

Licenciado y Profesor en Ciencias de la Educación. Facultad de Humanidades y Artes. Universidad Nacional de Rosario 
Union Secretary of our Trade Union of UNR Teachers and Researchers, COAD, (between 2005 and 2007), and then as General Secretary (between 2007 and 2009), prompted the need to systematize in an approximate way, a set of conceptual contributions built from the theoretical and methodological point of view of an ethnographic field work. This paper aims to provide an introduction into the identification of some of the changes taking place in the process of teaching work at University based on the new regulations introduced by the neoliberal model demanded by the World Bank for higher education that have been modifying the notion of teaching work" itself, its organization and the academic culture", affecting the subjectivity of the university teacher as worker, in their triple condition of wage-earner, professional and intellectual displayed in the context of mercantilization and the alienation of knowledge.

\section{Key Words}

Work - Subjectivity - Culture - Flow - Complexity

\section{Introducción}

Para quienes apostamos a una universidad que no contraponga calidad y eficiencia a la recuperación del sentido popular del espacio público y el pensamiento emancipador, la preocupación por lo que se naturaliza al interior de nuestras casas de estudio como una suerte de flujos (ver Foucault, 1993 y Deleuze y Guattari, 1995) de fuerzas, tendencias, saberes y discursos sin sujeto aparente, junto con la disolución de proyectos colectivos que tiene expresión concreta incluso en los pequeños equipos de cátedra; se revela como un estado de situación que nos reclama profundizar teóricamente y reelaborar conceptualmente en materia de política educativa desde la constatación y contrastación de lo que las Ciencias de la Educación pretenden aportar a los nuevos diseños que nos demandan la universidad y el mercado educativo de las competencias, en nombre de la sociedad y su progreso.

\section{La investigación y el papel formativo de los sindicatos docentes universitarios en el análisis de las modificaciones del proceso de trabajo}

Espacios de investigación impulsados a instancias de algunos sindicatos de UU.NN. que venimos compartiendo experiencias de formación, e intercambiando equipos de capacitación -como es el caso de ADU San Luis, AGD Río Cuarto, COAD Rosario y ADIUC Córdoba- nos han permitido iniciar una suerte de de-construcción de nuestros procesos de trabajo, desandando a partir de preguntas claves, los modos en que los procesos de evaluación instalados en la década del ' 90 organizan y construyen disciplinamientos en nuestras labores. Situación que a pesar de fuertes enunciados ideológicos construidos a lo largo 
de nuestra historia y nuestro compromiso político, marcó tendencias claras en los procesos de subjetivación (Bleichmar, 2005) de los docentes universitarios en su condición de tales poniendo en tensión y discusión, toda teoría construida alrededor de una recurrente concepción epistemológica de sujeto sustancial llamado a cumplir con su misión histórica sin fisuras ni contradicciones (aún en aquellos que se autoafirman ideológica y teóricamente, en la idea de que la contradicción constituye el motor de la historia).

Investigaciones como la encabezada por la Lic. Ana María Tello en el año 2000 (ver Tello, 2008) y desplegada en el ámbito del gremio docente de San Luis, comenzaron a develar -al interrogarnos por las políticas de evaluación con esta cultura de la pedagogía del control instalada en las universidadescómo y hasta qué punto se habían ido modificando las prácticas cotidianas y los modos de ser docentes. Empezaron a reconocerse así, desplazamientos significativos asociados a un nuevo marco legal que por medio de ordenanzas que regulaban la carrera docente y otros tantos reglamentos, condicionan las propias relaciones en el trabajo.

Es de hacer notar que tomamos aquí el concepto de cultura en la perspectiva con que lo hace García Canclini (1986), como un tipo especial de producción de fenómenos que contribuyen mediante la representación y elaboración simbólica a comprender, reproducir y transformar el sistema social.

El ejemplo más notable de lo registrado, lo constituye el Programa de Incentivos y Categorización en la Investigación, los que produjeron severos estallidos del cuerpo docente como colectivo, pero también, atacando el propio cuerpo de cada docente dejando marcas subjetivas que se evidenciaban a partir de la redefinición de los vínculos por medio de formas elitistas claramente competitivas, en una carrera meritocrática contra el tiempo y las formas de producción teórica con evidencias de marcas físicas en la salud, por la propia calidad de vida y condiciones de trabajo frente a la inestabilidad laboral y la precarización universitaria. Una nueva dinámica fue reconfigurando el proceso de trabajo. Proceso del que además, debemos recuperar (en palabras de Karl Marx en El Capital, Proceso de trabajo y proceso de valorización ) su definición de actividad orientada a un fin, el de la producción de valores de uso por medio de la articulación de varios elementos simples y abstractos: la propia actividad personal del trabajador (el trabajo mismo); el objeto sobre el cual ejerce esa actividad; y los medios a través de los cuales la ejerce (útiles, maquinarias, instalaciones, sistema de organización, etc.); y al que algunos autores como Jacques (ver por ejemplo, Perrin, 1980), incorporan el aporte de energía (que puede provenir de diferentes fuentes) y la provisión y procesamiento de un cierto volumen de información. Y en este punto también, volver a recuperar su diferenciación respecto del proceso de valorización, en lo que constituye la producción de mercancías que se instala como resultado de la unidad de proceso de trabajo y la formación del valor (valor de cambio de la fuerza de 
trabajo). Valor establecido por la cantidad de trabajo socialmente necesario para esa producción.

Cuestiones como la meritocracia y el individualismo (que ya anidaban en nuestro espacio social) emergieron con fuerza inusitada. La docencia quedó devaluada y marginada como tarea y proceso de elaboración detrás de títulos que se ponían de manifiesto ostentando más una suerte de carácter nobiliario, que por la responsabilidad de su carga laboral, sus funciones y sus condiciones al frente de los equipos de cátedra. La información que brindan las entrevistas en nuestro espacio sindical, habla de lo silenciado y naturalizado en el espacio académico que se expresa en términos de que, el que hace investigación, realiza un trabajo que vale y se pone de manifiesto en un riguroso sistema de papers y acreditaciones. Y el que hace docencia, queda invisibilizado porque más allá de la energía depositada y el desgaste de su fuerza de trabajo, no se estipula lo que es necesario observar y no califica.

Del mismo modo, Martínez (1997, 2008), sostiene que cada vez que se empieza a mirar el trabajo de los docentes en cualquiera de sus niveles, la llamada al psicólogo es por el sufrimiento psíquico, por el nivel de padecimiento que ha traído este modo de trabajar. Y que la respuesta a cómo salir de esta situación, no la pueden encontrar ni siquiera con los que sufren, aunque sean los más apurados y necesitados de salir del dolor. $Y$ uno se pregunta por qué estás aguantando eso todo el tiempo. Ella nos alertaba de la necesidad imperiosa de entrar en el meollo de un problema práctico: ¿cómo se trabaja en la universidad? Pero incorporando el conjunto de todos los que trabajan, incluyendo a los estudiantes, no solo a los asalariados. Y que esto es fundamental para poder investigar y meternos más profundamente en el proceso de trabajo como categoría de análisis. Que si seguimos en esta línea, podemos ir avanzando en el sentido de encontrar uno de los núcleos duros del problema. Porque hoy hay un hueco, un déficit, y hay que estudiarlo para poder tener propuestas hacia la exploración y elaboración de las nuevas regulaciones. Martínez (2008) nos lo advertía con la frescura de toda su experiencia, que estas (las propuestas) "no salen solamente de la militancia y el discurso académico o político, con frecuencia, vacios de contenido. En realidad este análisis puede hacerse tomando posición desde muchos ángulos, y uno, es poder ingresar en la subjetividad de los trabajadores y de los estudiantes" (p. 136).

Desde nuestra intervención sindical, partimos de ubicar en primera instancia y sin ambages al docente universitario como trabajador, en la perspectiva ampliada con que Antunez (2005) habla de clase trabajadora, enmarcándonos además de en el plano de la producción, reproducción, circulación y transferencia de saberes, en el contexto de la expresión clase-que-vive-del-trabajo, con el objetivo de otorgar cierta validez contemporánea a la noción de clase. Particular y especialmente, cuando actuales formulaciones teóricas plantean la pérdida de validez analítica e incluso el supuesto fin del trabajo, con la centralidad con 
que lo ubica el pensamiento marxista. La intencionalidad de su planteo, es dar contemporaneidad y amplitud al ser social que trabaja, la clase trabajadora de hoy. Aprehender su carácter efectivo, su carácter procesual y su forma concreta. No se restringe al trabajo manual directo, sino que incorpora la totalidad del trabajo social, la totalidad del trabajo colectivo asalariado. Incorpora formas de trabajo que son productivas, que producen plusvalía, pero que no son directamente manuales. Suma, al trabajo productivo con centralidad en el proletariado industrial como su núcleo principal, a los trabajadores improductivos. Aquellos cuya forma de trabajo es utilizada como servicio, ya sea para uso público o para el capitalista y que no se constituyen como elemento directamente productivo, como elemento vivo del proceso de valorización del capital y de la creación de plusvalía. Son aquellos en quienes, según Marx, el trabajo es consumido como valor de uso y no como trabajo que crea valor de cambio. Y abarca un amplio abanico de asalariados, desde aquellos insertos en el sector de servicios, bancos, comercio, turismo, servicios públicos, etc., hasta aquellos que realizan actividades en las fábricas pero que no crean valor en forma directa. Desde esta perspectiva y siguiendo esa lógica es que nos permitimos introducirnos en el campo de las mutaciones de nuestro trabajo docente, o de manera más abarcativa, el trabajo mismo que realizamos.

Podemos comprobar hoy que la posición de privilegios no pasa sólo por la acumulación de dinero, sino también por la apropiación del saber, por supuesto, científico. Y precisamente el saber puede llevar en sí apropiación de riquezas. Y no cabe dudas de que es así. Ante esta evidencia, surgen diversas tendencias desde el movimiento emancipador, desde muchos pensadores y sujetos sociales preocupados -como nosotros- por la renovación del marxismo como teoría de liberación, que proponen redirigir la lucha de clases hacia la disputa de esos conocimientos. Se argumenta que, siendo hoy el conocimiento -el conocimiento científico- el factor principal de producción de riqueza, el detentador de dicho conocimiento sería el actual sujeto, como ayer lo fue el productor manual. Esta línea de pensamiento no logra superar la noción de un sujeto sustancial, que deviene desarrollo de las fuerzas productivas, y por lo tanto, nos llevaría a un nuevo callejón sin salida (Mattini, 2004, p. 126).

La cita resulta interesante en la medida que nos preocupan los procesos de subjetivación del sujeto, en su condición (la nuestra) de trabajador docente universitario que administra -en su relación con estudiantes y colegas- saberes y conocimientos validados científicamente, pero que se lo soslaya en su posibilidad (la nuestra) de calificar por su condición de trabajador en el espacio pedagógico del área de conocimientos o de la disciplina que fuere, más allá de las áreas prioritarias de las que se trate. Y junto a esto, un sistema de jerarquías que no tienen respaldo ni control por convenio colectivo alguno (que 
no casualmente los rectores en el Consejo Interuniversitario Nacional, CIN, se niegan sistemáticamente a discutir), rebajando el sentido de la organización laboral y la valoración de la producción de conocimientos, mientras otro conjunto reducido de colegas devenidos funcionarios de ocasión por tareas de gestión, vuelcan sus labores hacia una verdadera carrera de funcionarios en la que se desplazan progresivamente hacia formas patronales de administrar la política educativa en la educación superior consolidando un modelo de universidad aconsejado por el Banco Mundial, que Slaughter y Leslie (1977) han denominado Capitalismo Académico, a partir de constituirse las universidades como áreas de negocios dignas de ser explotadas.

No es menor entonces, en cuanto a sus efectos en quienes la constituimos y sostenemos diariamente, el profundo cambio de perspectiva con que es pensada y concebida la universidad pública más allá de los discursos de quienes en una suerte de doble moral buscan su propia legitimidad política con reiteradas apelaciones a la Reforma Universitaria de 1918, la autonomía universitaria (concepto hoy profundamente discutido) y la democracia (que no logra desprenderse de su matriz política histórica de corte liberal-conservador).

Como bien lo plantea Campos:

La universidad había sido comprendida como referente cultural y reflexivo básico de la sociedad, espacio de diálogo y discusión propio de la racionalidad moderna fundado en el accionar de sus académicos, vistos como órganos permanentes de una institución fundamental de la sociedad moderna... La universidad, por lo anterior, fue caracterizada por su solidez e inmanencia, las generaciones pasan y la universidad permanece durante cientos de años como espacio de reflexión crítica de la sociedad [...] La universidad como institución fundamental, unidad social relativamente estable y permanente, de límites claros y perennes, solidez para la reflexión certera de la sociedad moderna, solemnidad garantizada para la celebración de la razón. Esto es lo que menos caracteriza a la universidad que hoy comienza a gestarse y gestionarse en diversos rincones del planeta adquiriendo paulatinamente el estatuto propio de las empresas que prestan algún servicio a la sociedad para pasar a ser tan sólo una organización del mercado, en el contexto de un proceso de reordenamiento coherente con las lógicas liberales que guían la lógica organizativa de la universidad al autofinanciamiento, orientando la producción de saberes y técnicas según las demandas de una sociedad, comprendida como capacidad de consumo (Campos, 2005, p. 524).

Es necesario entonces, penetrar en el estudio de las mutaciones que han vivido las universidades tomando como objeto de estudio a los docentes universitarios en condiciones de precariedad laboral, porque son ellos los que mediante vinculaciones flexibles y bajo el amparo de ninguna legislación laboral, 
llevan a cabo la vida de la universidad de flujos, la universidad flexible, capaz de articularse y reordenarse según las demandas de la sociedad/mercado. La flexibilización laboral encuentra al docente universitario del modelo neoliberal, que hoy se proyecta con más continuidades que rupturas en estos pretendidos tiempos post-neoliberales con ciertos perfiles neodesarrollistas impulsados con particular énfasis desde el 2003 a la fecha, como un trabajador precario. Sometido a la precarización de sus condiciones contractuales y ocupacionales en general, es decir, las físicas y materiales propias del puesto de trabajo y de su vinculación social a la organización, ubicándonos como meros prestadores de servicios. Los servicios que prestamos son horas de docencia. Y se busca maximizar el recurso del trabajo docente como capital humano de la universidad, reduciendo sus costos y aumentando los beneficios sobre el proceso productivo en el cual se sustenta cada universidad.

La teoría del capital humano considera que el gasto en educación de un individuo consiste en dos componentes, uno de consumo y otro de inversión (Pescador, 1994). Y que todo se basa en una teoría de la elección racional y maximización de las utilidades bajo ciertas restricciones. De aquí surge el concepto de tasa de retorno a la inversión en la educación y que según Torres (2001), desde que se diseminaron en los ambientes burocráticos-educativos de América Latina han constituido una referencia ineludible en las toma de decisiones educativas.

Cabe observar en el análisis teórico-político-pedagógico (ver Rinesi, 2008) que uno de los sitios donde en gran medida se expresan esas continuidades mencionadas, es en los propios comportamientos corporativos de los actores del sistema universitario, e incluso las propias cabezas -las propias subjetividades- de los universitarios.

Esa ha sido la gran operación política que introdujeron los '90 en la organización universidad, extendiéndose hasta nuestros días: un disciplinamiento transformado progresivamente en cultura académica construida sobre la fragmentación y debilitamiento de los lazos y los anclajes sociales de los cuerpos docentes, devenidos estos (los trabajadores docentes) ejecutores de las políticas concebidas por los expertos. Se consolida así (como ya lo marcáramos en anteriores trabajos) la fractura entre concepción y ejecución, y sistemas de evaluación según estándares del mercado global del conocimiento en el que los sectores políticos hegemónicos que gestionan la universidad sobre la base del vaciamiento de sentido y profundidad del debate académico y político de Consejos Directivos, Consejos Superiores y Asambleas Universitarias, defienden inversiones -y por qué no, negocios-, más que proyectos. Y he aquí la centralidad que pueden adquirir nuestros sindicatos docentes respecto a cómo intervenir con la mayor autonomía posible en el debate, la formación y capacitación docente, frente a las políticas educativas y culturales que se articulan entre el CIN y el Ministerio de Educación de la Nación, extendiéndose 
también al interior de célebres cátedras teñidas de bronce que operan como ilustres paradigmas.

\section{Alfa Tuning, "nuestro" proceso de Bolonia y el mercado de competencias}

Mientras Rama (2006) nos alertaba en su libro La Tercera Reforma de la Educación Superior en América Latina, de la marcha hacia una nueva dinámica de un modelo público-privado-trasnacional y una nueva figura en el sistema universitario de la región, que comienza a cumplir el rol de policía educativa al establecer niveles mínimos de calidad, criterios de autorización de funcionamiento de las instituciones y otras diversas regulaciones a la libertad de competencia (los SACES, Sistemas de Aseguramiento de la Calidad de la Educación Superior -organismos conformados en la mayoría de los casos por representantes de los diversos actores académicos, con alto grado de autonomía y que no dependen directamente de los ministerios de educación-); en el 2005, el actual Senador y entonces ministro de Educación, Ciencia y Tecnología, Daniel Filmus, y su -en ese momento- Secretario de Políticas Universitarias, Dr. Juan Carlos Pugliese, presidían la presentación del Proyecto Alfa Tuning para América Latina.

Uno de los objetivos centrales era (sigue siendo, impulsado por el actual Secretario de Políticas Universitarias, Alberto Dibbern, y el CIN que lo ha retomado dando nuevo empuje) el de

[...] contribuir al desarrollo de titulaciones fácilmente comparables que faciliten la movilidad de los poseedores de títulos universitarios y profesionales en América Latina. Se trata de alcanzar un amplio consenso a escala regional sobre qué actividades tienen capacidad de desempeñar los diferentes profesionales. De este modo, se buscan puntos comunes de referencia centrándose en las competencias y destrezas, siempre basadas en el conocimiento (Secretaría de Políticas Universitarias, 2010).

Ellos afirmaban que el proyecto ha sido diseñado como un proyecto independiente, impulsado y coordinado por universidades (públicas y privadas) de distintos países, tanto latinoamericanos como europeos. Se expresa además que

[...] el Proyecto Tuning surge en un contexto de intensa reflexión sobre educación superior que se ha impuesto como consecuencia del acelerado ritmo de cambio de la sociedad. El proyecto está especialmente vinculado a la experiencia llevada adelante por más de 100 universidades europeas que desde 2001 vienen trabajando en conjunto. Sus objetivos se enmarcaron en el proceso de BoloniaPraga-Berlín, a través del cual los políticos aspiran a crear un área de educación superior integrada en Europa en el trasfondo de un área económica europea (SPU, 2010). 
En ese contexto, y analizando las modificaciones en el mercado de trabajo y los usos de la revolución tecnológica, Cardelli (2008) expresa que así como en los '90 nos hicieron subir al tren de la modernidad menemista, los especialistas de las nuevas tendencias del mundo globalizado nos están diciendo que debemos subirnos al tren de esas tendencias que se generan en los centros capitalistas y que expresan las transformaciones de la educación superior en una suerte de eurocentrismo de los procesos culturales, y que Argumedo (2001) lo planteó en términos de vivir y organizar nuestras vidas y nuestras culturas a partir de lo que piensan los países centrales. Sólo que, nos dice Cardelli (2008), las tendencias son una realidad, pero tienen sujetos que las impulsan y que son los que se benefician. En su interior expresan el movimiento dialéctico de la realidad. No son tendencias abstractas, sino expresiones del desarrollo del modelo de producción capitalista en esta etapa en particular, que expresan los intereses del capital. Y por ese motivo es fundamental profundizar en torno a las características objetivas de la revolución científico-tecnológica actual y la llamada sociedad del conocimiento, ya que una de las consecuencias de esta revolución que todo lo invade, son las transformaciones del propio Estado, y la vida social y cultural, y por lo tanto la construcción de lo público y lo común.

En la misma mesa de discusiones, Rinesi (2008) nos observaba abriéndonos discusión en torno al desfinanciamiento de la universidad por el Estado (o el gobierno de turno y su política económica), que el tema es analizar en profundidad las decisiones acerca del modo de aplicar los muchos recursos que hoy se destinan. Y remarcaba la

[...] gran cantidad de dinero que el gobierno destina al sistema nacional de ciencia y técnica (y por esa vía, directa o indirectamente a las universidades) y que se expresan en el extraordinario aumento de los financiamientos a becas para las carreras de investigación, para el CONICET, para la Agencia Nacional, para programas como Ios PICT, los PICT-O, Ios PAV, etc. Muchísima plata sobre todo para la investigación asociada a la formación de postgrado (maestrías, doctorados, postdoctorados) [...] constituye la deformación del sistema universitario en una preocupante evidencia de la tendencia a la separación en el seno de nuestras universidades, entre la vida académica (que es la pobre vida de las carreritas por las titulaciones sucesivas de licenciado, master, doctor, pos doctor, pos-pos-pos... un absurdo correteo hacia la nada) y la vida intelectual que corre el serio riesgo de desaparecer si termina por triunfar esa pobre lógica carrerista de las universidades argentinas (Rinesi, 2008, p. 21).

Es para nuestros gremios una cuestión clave poner aquí el énfasis en la evidente y lamentable separación existente entre vida académica y vida intelectual. Hay un indicador que es la desaparición de los espacios de debate y formación vinculados a la historia y a la epistemología de la ciencia y la 
actualidad de los grandes problemas científicos. Hablamos de grandes en la primera preocupación por hallarlos, por definirlos y no permitir sin más, que se nos marque la agenda. Esta ausencia provoca una adscripción a cualquier tipo de producción teórica, con una única condición: que se publique en revistas con indexación. Pero tal vez, más serio aún, resulte la desvinculación entre los académicos y las organizaciones sociales (particularmente entre los académicos de las ciencias sociales).

Rinesi (2008) cerraba su razonamiento planteando que como este sistema, después de producir la tanda de masters y doctores que está produciendo, necesita seguir produciendo más masters y doctores y postdoctores, existe en el sistema universitario un fuerte estímulo para quienes se han recibido (cuanto antes, mejor) de masters, doctores y post doctores, para convertirse también rápidamente, en profesores de la gran cantidad de maestrías, doctorados y post doctorados que aparecen por todas partes, que son un gran negocio, y que pagan mejor y dan más prestigio que los cursos de grado regulares en los que también podrían trabajar. Y finalmente su intervención terminaba, afirmando que

[...] la distorsión es completa: chicos de 32, 33 años, con su doctorado recién obtenido, creen que -como me dijo días pasados uno de ellos- están para dar clases en el doctorado, y como eso les conviene, y como les da más puntos en la loca carrera hacia la nada en que han decidido convertir su vida, se ponen a dar clases en cuanto doctorado los reclame (y consiguen, por supuesto, trabajar muchísimo, porque esos doctorados se multiplican como hongos y los necesitan), a veces sin siquiera haberse formado antes como profesores en cursos de grado, porque con cada vez mayor frecuencia no tuvieron que trabajar de eso ni de nada hasta esa edad de sus vidas, que atravesaron con todo éxito saltando ágilmente de beca en beca [...] (Rinesi, 2008, p.26).

En nuestra opinión, y según datos chequeados que serían motivo de una publicación más extensa que el presente artículo, la inversión en ciencia y tecnología muy por el contrario es escasa. Lo que sí ha habido, es una fuerte inversión en los grupos hegemónicos en ciencia y tecnología. La concentración de la asignación de fondos para la investigación en manos del Ministerio y la Agencia, ha provocado el empobrecimiento de los presupuestos destinados a investigación en las UUNN. Esto implica concentración de fondos en pocos grupos y empobrecimiento de la mayoría de los equipos de investigación del país. 


\section{¿Cuáles competencias? ¿Sobre qué formación profesional y docente? ¿En el marco de qué proceso de trabajo?}

Junto al Trayecto Curricular Subjetividad y trabajo docente. Hacia un nuevo proyecto de universidad llevado a cabo en nuestro sindicato entre Abril y Setiembre de 2009, uno de los cursos de capacitación más importantes que desarrolláramos desde COAD, en combinación con la Escuela de Ciencias de la Educación y en el marco del mismo Programa Nacional de Capacitación Docente Gratuita, fue sin lugar a dudas el Seminario de Alicia de Alba de Abril de 2008, EL Campo del Currículum en la Primera Década del Siglo XXI. Tensión Globalización-Crisis Estructural Generalizada. Allí, de Alba nos introducía al concepto de posicionalidad en un esfuerzo por de-construir el discurso globalizador, sumergiéndonos en la complejidad de su trama. Nos advertía que por posicionalidad, se entiende al espacio ontológico, semiótico, epistémico, teórico, psíquico, cultural, social, geopolítico, económico desde el cual se enuncia, se construye la palabra. La posicionalidad del discurso le imprime a éste la fuerza del entramado social del cual forma parte el que enuncia, se refiere al espacio de la enunciación. Y nos planteaba que por eso mismo, es a la vez interesante e implica un compromiso político de estudiosos, académicos y ciudadanos, el confrontar el significante globalización con concepciones y datos para reconstruir el efecto de veracidad y totalidad.

E introduciéndonos de Alba en la sociología de las competencias (ver Diaz Villa, 2006), observábamos que se trata de una realidad constituida y constituyente (estructurada y estructurante) en el sujeto que le permite desempeñarse en diferentes niveles, planos y contextos, más allá de parámetros, lógicas establecidas y de límites impuestos. Y asumir por lo tanto, modos de aprender, pensar y hacer -actuar- en la sociedad. Que nominalmente, esa realidad la definimos como la competencia profesional, un objeto de conocimiento que es el producto de una combinación de condiciones y posibilidades objetivas y subjetivas. Díaz Villa (2006) remarca que, condiciones objetivas, porque

a) La competencia -como una construcción social- no puede abstraerse de los procesos y relaciones que la construyen, jerarquizan y legitiman.

b) La competencia se desarrolla y valida en instituciones especializadas cuyas ideologías y proyectos formativos están delineados y expresados en currículos y pedagogías específicas.

c) Dichos procesos formativos se realizan en el interior de campos de conocimiento y de práctica fundamentados en principios, teorías, hipótesis, interpretaciones, explicaciones y argumentos, que se comparten y que se consideran legítimos. En este sentido, el conocimiento profesional es un producto colectivo, social que, definido como legítimo, comparten los profesionales de una comunidad epistémica. 
d) Se configuran en prácticas de interacción (enseñanza-aprendizaje) en contextos específicos donde se refuerza la actividad y creatividad del estudiante, en relación con los saberes y prácticas propios del campo.

e) Su logro y desarrollo está asociado a fines y objetivos relacionados con el saber y el hacer dentro de contextos específicos (o, de manera transversal, a la relación entre contextos), y a criterios de actuación o desempeño verificables y demostrables.

$Y$ condiciones subjetivas porque:

a) La competencia se asocia de manera intrínseca a los sujetos, quienes la poseen y desarrollan.

b) El conocimiento es un substratum básico de la competencia, que acontece en la mente del hombre.

c) La competencia se asocia a la manera como el sujeto se relaciona con objetos de conocimiento o aprendizaje.

d) Presupone ciertas facilidades intrínsecas a todos los individuos, que pueden considerarse semióticamente como una gramática interna.

e) Se relaciona con rasgos y factores subjetivos de los individuos, en nuestro caso los futuros profesionales.

Díaz Villa (2006) reafirma el sentido de su análisis, planteando que la competencia ha sido definida de diferentes maneras dependiendo del contexto en el cual se requiera la noción. Apropiando a Bernstein (1998) es posible decir que la noción de competencia ha entrado en el juego de la exportación o de la recontextualización. Esta ha producido una pluralidad de significados cuya utilidad depende de intereses bajo los cuales se elabora una u otra definición. En este sentido, podemos argumentar que la mayoría de las definiciones de la competencia, especialmente de aquellas que se refieren a lo que se ha dado en denominar competencias profesionales, son descripciones teóricamente débiles que operan con objetos extrínsecos, por ejemplo una habilidad, los rasgos de un desempeño, las características de un oficio, una acción, etc. En cierta forma, los significados de competencia en estos campos dependen de las descripciones e interpretaciones que se tengan. Dicho de otra manera, los principios de descripción actúan selectivamente sobre lo que se convierte en significado, en este caso, de competencia. Este anclaje de la definición a partir de los principios de descripción, no permiten establecer cómo se constituye una competencia, cómo un desempeño se traduce en competencia y viceversa.

Resulta imprescindible destacar que sobre la noción de competencia que atraviesa al Programa de Bolonia y el Alfa Tuning, se monta lo que -a nuestro juicio y el de no pocos estudiosos del tema- constituye uno de los dispositivos de exclusión más fuertes. Además, está sobradamente analizado y comprobado que las competencias no son enseñables, se desarrollan en los procesos de trabajo y cuando éstos pueden ser llevados al plano del análisis del sujeto que 
lo realiza (a veces se comparte con el otro/s, y otras, queda en la subjetividad más profunda...ni el mismo sujeto sabe que tiene tal o cual competencia. Por otra parte, no es asimilable a la necesidad de enseñar y fijar claramente contenidos conceptuales, actitudinales y procedimentales, como indicaba por ejemplo la Ley Federal de Educación).

\section{Algunas reflexiones finales de carácter iniciático}

Si la explotación económica separa la fuerza y el producto del trabajo, digamos que la coerción disciplinaria establece en el cuerpo el vínculo de coacción entre una aptitud aumentada y una dominación acrecentada (Foucault, 1991, p. 245).

Morín (1990), en su Introducción al pensamiento complejo, señalaba que Bachelard, el filósofo de las ciencias, había descubierto que lo simple no existe, sólo existe lo simplificado. La ciencia construye su objeto extrayéndolo de su ambiente complejo para ponerlo en situaciones experimentales no complejas. Que la ciencia no es el estudio del universo simple, que es una simplificación heurística necesaria para extraer ciertas propiedades y ver ciertas leyes. Y al mismo tiempo proponía que la acción supone complejidad, es decir, elementos aleatorios, azar, iniciativa, decisión, conciencia de las derivas y de las transformaciones. Que es por eso que tenemos que utilizar múltiples fragmentos de acción programada para poder concentrarnos sobre lo importante. No hay un dominio de la complejidad que incluya el pensamiento, la reflexión, por una parte, y el dominio de las cosas simples que incluiría la acción, por la otra. La acción es el reino concreto y, tal vez, parcial de la complejidad.

Nuestra acción sindical, asumiendo ese carácter, ha tenido la osadía de pretender internarse en la complejidad de los procesos de trabajo de un metamorfoseado nosotros docentes universitarios, que se permita razonar desde una discontinuidad epistémica. Un nosotros que ha sufrido un largo proceso de desconfiguración con corrimientos y desplazamientos en un profundamente modificado campo simbólico de jerarquías, responsabilidades, dedicaciones, capacidades y competencias en nuestra condición de trabajadores precarios, evaluados con indicadores propios de la tarea de investigación, y no de docencia. Aflora entonces que cada docente y cada grupo y equipo de docentes fue indirectamente disciplinado, confinándolos-nos a un pequeño fragmento en el desmanejo global de la totalidad de las variables intervinientes que inciden en, y caracterizan la administración de nuestro propio campo disciplinar. Lo que produjo marcas objetivas en la subjetividad individual y colectiva con una escisión que refuerza la enajenación de la construcción social de conocimiento en la que afirmamos instalarnos, distanciándonos así de nuestro propio producido. 
Si como sostiene Foucault (1991), la organización de un espacio serial fue una de las grandes mutaciones técnicas de la enseñanza elemental, que permitió sobrepasar el sistema tradicional y al signar lugares individuales hizo posible el control de cada cual y el trabajo simultáneo de todos organizando una nueva economía del tiempo de aprendizaje y haciendo funcionar el espacio escolar como una máquina de aprender, de vigilar, de jerarquizar, de recompensar; pues bien, la desconfiguración de este nosotros universitarios producidos por las leyes educativas de los '90 en el marco de la revolución científico-tecnológica en la era digital de las comunicaciones, nos marginó con un efecto centrífugo y confinó con un mismo sentido disciplinador y colonizante, recluyéndonos en la desconfianza hacia el otro diferente y en una búsqueda por sobrevivir en el pequeño espacio propio asociado en soledad al recurso tecnológico.

Se trataría entonces de aportar desde las ciencias de la educación, a descolonizar nuestro sistema de pensamiento, incluso cierta racionalidad pretendidamente crítica y analítica, que introduzca una lógica de ruptura como corte a la ilusión del continuismo o la perpetuación de un estado lineal de flujos y tendencias -como modo de ser y constituir- ese nosotros docentes universitarios, que pueda a la vez, abrir espacio efectivo a la creación intersticial. Para que por medio de la recuperación de la autoría de la palabra-pensamiento de, y en, nuestros procesos laborales, establezcamos una nueva posibilidad de reinventar nuestro vínculo con el conocimiento y con quienes trabajamos con él hacia la emancipación social que postula Boaventura de Sousa Santos (2008), cuando dice que nunca la disonancia, la discrepancia entre teoría crítica y teoría política de emancipación, y práctica de emancipación, ha sido tan grande como ahora.

Los años '60 fueron marcados en el debate teórico-político, por una fuerte crítica desde el pensamiento emancipador hacia el desarrollismo y el estructuralismo -como corrientes hegemónicas- en sus lógicas justificadoras de los poderes dominantes con una pretendida perspectiva progresista del desarrollo social. Aún a riesgo de caer en una mirada reduccionista, es posible que nos encontremos a las puertas de una situación similar, cuando se instala esta perspectiva pública-privada-transnacional del conocimiento mercantilizado y concentrado en especialistas por competencias como si esta fuera la llave mágica para alcanzar la equidad social, con un pacto nacional que anule el conflicto equiparando discursivamente las nociones de progreso, crecimiento y desarrollo.

Este es el punto en el que es bueno revisar los enfoques de Norberto Bobbio, cuando caracteriza de tres maneras diferentes, las aplicaciones del concepto de desarrollo al analizar el desarrollismo: 1) Como mero crecimiento. Sostenido por los ortodoxos neoliberales y para los que el macro-crecimiento de la macro-economía es lo que finalmente importa al modelo, con lo que la economía familiar y el bienestar para todos no pasan de ser meras consignas. 
2) Como proceso. Entendido en una perspectiva mecanicista de simple sucesión de fases. Así los países, al margen de las determinaciones de los grandes intereses económicos mundiales, estarían destinados a transitar naturalmente del subdesarrollo a una sociedad moderna gracias al impacto de las leyes del mercado. 3) Como perspectiva del cambio de estructuras. Llama la atención sobre las causas, determinaciones y obstáculos estructurales para lograr el desarrollo. Con lo que sin atender este nivel, el desarrollo resulta imposible. Pero justamente, habría aquí que observar la impronta estructuralista, que deviene fuertemente economicista y mecanicista.

Los llamados tiempos post-neoliberales en condiciones de precariedad laboral frente al conocimiento, constituyen entonces el desafío académico y sindical del pensamiento político-pedagógico de un cada vez más necesario movimiento de trabajadores docentes universitarios en cada uno de nuestros campos disciplinares e institucionales. Entiendo, y así lo hacen no pocos compañeros docentes con los que compartimos las experiencias mencionadas, que uno de los núcleos que debiéramos volver a analizar, es la existencia o actual fortalecimiento del sector de la universidad que podríamos denominar nuevo profesionalismo. Nos referimos a quienes tienen fuerte presencia política al interior de las UU.NN. pero que viven de sus ingresos del afuera. Particularmente (no sólo...) parte de los abogados, contadores, ingenieros, agrónomos, etc., y que dudamos acuerden hoy con una política de aumentos de dedicación y estén comprometidos en una política de aumentos salariales vinculada a los procesos laborales analizados. Si actualizáramos a Varsavsky, tal vez el lugar del cientificismo lo ocuparía este nuevo sector.

[...] Resumiendo: cientificista es el investigador que se ha adaptado a este mercado científico, que renuncia a preocuparse por el significado social de su actividad, desvinculándola de los problemas políticos, y se entrega de lleno a su carrera" aceptando para ella las normas y valores de los grandes centros internacionales, concretados en un escalafón (Varsavsky, 1994, p. 54).

\section{Referencias Bibliográficas}

- ADIUC-AGD-COAD. (2008). Reflexiones del Congreso Nacional por una Educación Superior Democrática, Popular, Emancipadora y Latinoamericana. Hacia la construcción de un nuevo movimiento nacional de trabajadores docentes universitarios. Córdoba: GraficArce.

- Antunes, R. (2005). Los Sentidos del Trabajo. Ensayo sobre la afirmación y la negación del trabajo. Buenos Aires: Herramienta Ediciones. TEL-Taller de Estudios Laborales.

- Argumedo, A. (2001). Los Silencios y las Voces en América Latina. Notas sobre el pensamiento nacional y popular (3 ed.). Buenos Aires: Ediciones del Pensamiento Nacional. 
- $\quad$ Bleichmar, S. (2005). La subjetividad en riesgo. Buenos Aires: Topía.

- Bobbio, N.; Matteucci, Nicola; Pasquino, Gianfranco; Aricó, José; Soler; Tula, Jorge. (1991). Diccionario de Política. (6 ed., 2 Tomos). Buenos Aires: Siglo XXI.

- Campos V.S. (2005). Flexibilización laboral de la docencia universitaria y la gest(ac) ción de la universidad sin órganos. Un análisis desde la subjetividad laboral del docente en condiciones de precariedad en Gentili. P. y Levy, B. (comp.). Espacio público y privatización del conocimiento. Estudios sobre políticas universitarias en América Latina. Buenos Aires: CLACSO.

- Cardelli, J. (2008). Las modificaciones en el mercado de trabajo y los usos de la revolución tecnológica en Reflexiones del Congreso Nacional por una Educación Superior Democrática, Popular, Emancipadora y Latinoamericana. Hacia la construcción de un nuevo movimiento nacional de trabajadores docentes universitarios. Córdoba: GraficArce.

- Deleuze, G. y Guattari, F. (1995). Mil Mesetas - Capitalismo y Esquizofrenia. Madrid: Pre-Textos.

- Díaz Barriga, A. (1994). Docente y Programa. Lo institucional y lo didáctico. Buenos Aires: Aique.

- Díaz Villa, M. (2006). Hacia una sociología de las competencias. Mimeo. Santiago de Cali.

- $\quad$ Foucault, M. (1991). Vigilar y Castigar. Nacimiento de la prisión (7 ed.). Buenos Aires: Siglo XXI.

- $\quad$ Foucault, M. (1993). Microfísica del poder. Madrid: La Piqueta.

- García Canclini, N. (1986). Las culturas populares en el capitalismo. México. Nueva Imagen.

- Guyot, V. (1998). Universidad e Investigación en Argentina. Acerca de lo que nos reveló la crítica al "cientificismo". Alternativas, 10, 113.

- Martínez, D., Iris, V. y Kohen, J. (1997). Salud y Trabajo Docente. Tramas del malestar en la escuela. Buenos Aires: Kapelusz.

- Martínez, D. (2008). Salud y Trabajo Docente, en ADIUC-AGD-COAD, Reflexiones del Congreso Nacional por una Educación Superior Democrática, Popular, Emancipadora y Latinoamericana. Córdoba: GraficArce.

- Mattini, L. (2004). El Encantamiento Político. De revolucionarios de los '70 a rebeldes sociales de hoy. Buenos Aires: Peña Lillo - Ediciones Continente.

- Mclaren, P. (1994). Pedagogía Crítica, Resistencia Cultural y la Producción de Deseo. Buenos Aires: Aique.

- Morin, E.(1998). Introducción al Pensamiento Complejo. Barcelona: Gedisa.

- Neffa, J. C. (1990). El Proceso de Trabajo y la Economía de Tiempo. Buenos Aires: Humanitas.

- Rama, C. (2006). La Tercera Reforma de la educación superior en América Latina. Buenos Aires: Fondo de Cultura Económica de Argentina.

- Requejo, M. I. (2009). Lingüística Social y Autorías de la Palabra y el Pensamiento. Temas de debate en Psicología Social y Educación. Buenos Aires: Ediciones Cinco.

- Rinesi, E. (2008). Sistema de Educación Superior en ADIUC-AGD-COAD. Reflexiones del Congreso Nacional por una Educación Superior Democrática, Popular, Emancipadora y Latinoamericana. Hacia la construcción de un nuevo movimiento nacional de trabajadores docentes universitarios. Córdoba: GraficArce.

- Secretaría de Políticas Universitarias. Proyecto Alfa Tuning América Latina. Extraído el 15 de agosto de 2010 desde http://www.me.gov.ar/spu/guia_tematica/ENCIU/ enciu pyp alfa_tuning.html 
- Slaughter, Sh. And Leslie, L. (1977). Academic Capitalism: Politics, Policies \& the Entrepreneurial University. Baltimore: The Johns Hopkins University Press.

- Tello, A. M. (2008). Nuevas regulaciones en el trabajo docente, en ADIUC-AGD-COAD, Reflexiones del Congreso Nacional por una Educación Superior Democrática, Popular, Emancipadora y Latinoamericana. Córdoba: GraficArce.

- Torres, C. A. (Comp.) (2001). Paulo Freire e a agenda da Educaçao Latino Americana No Século XXI. Buenos Aires: Clacso

- $\quad$ Nuñez Hurtado, C. (2001). La Revolución Ética. "¡A nosotros no se nos ha muerto la esperanza!". Buenos Aires: Lumen Humanitas.

- Varsavsky, O. (1994). (8 ed.). Ciencia, política y cientificismo. Buenos Aires: CEAL.

- Yañes Cortés, R. (1991). Ruptura Epistemológica. Paradoja de la llusión-des-llusión. Análisis filosófico. Buenos Aires: Catálogos 